\title{
Telepsychiatry: new perspectives and open issues
}

\author{
Marta Valdagno, ${ }^{1 *}$ Arianna Goracci, ${ }^{1,2}$ Silvia di Volo, ${ }^{2}$ and Andrea Fagiolinil,
}

\footnotetext{
${ }^{1}$ Department of Mental Health, University of Siena Medical Center (AOUS), Siena, Italy

${ }^{2}$ Division of Psychiatry, Department of Molecular Medicine, University of Siena School of Medicine, Siena, Italy
}

Telepsychiatry, thanks to the continuous advance of technologies, is an area with excellent prospects to become an effective tool for assistance in psychiatry. Many studies evaluated the effectiveness of telepsychiatry in different psychiatric disorders like anxiety disorders, psychotic disorders and depression. Results showed that telepsychiatry seems to be promising in anxiety and depressive disorders, especially in the maintenance phase, and also for psychotic disorders, especially for these cases with a poor compliance. We reviewed the literature to evaluate the feasibility of telepsychiatry in Italy in terms of satisfaction, costs and cultural acceptability and considering ethical and legal aspects. In Italy the development of telemedicine still presents a high level of fragmentation. A potential integration of these new types of services with the health care system is a very challenging task and poses many legal and ethical challenges. Therefore the creation of legal instruments and formal professional ethical guidelines are needed.

Received 5 September 2013; Accepted 1 November 2013; First published online 2 January 2014

Keywords: Anxiety disorders, depression, psychotic disorders, telepsychiatry.

Telepsychiatry has been defined as "the delivery of health care and the exchange of health information for purposes of providing psychiatric services across distances." ${ }^{1}$ The increasing interest in this area is due to technological advances, which have made equipment less expensive and easier to use, as well as to rising healthcare costs, which mandate new ways of providing affordable, yet high quality, healthcare.

Telepsychiatry improves access to mental health care, for instance in the case of patients living in underserved areas, individuals with limited mobility, families with children, and patients who are homebound.

High levels of satisfaction have been reported, for example, in jail populations, ${ }^{2}$ rural settings, ${ }^{3}$ child and adolescent patients, ${ }^{4,5}$ geriatric patients, ${ }^{4,5}$ and patients with limited access to health care. ${ }^{6}$

Telepsychiatry is not intended as a substitute for traditional face-to-face therapy. However, it may provide an array of adjunctive services, including-but not limited to-diagnosis, assessment, and medication management, as well as individual and group therapy.

The efficacy of telepsychiatry seems to be very promising in the treatment of anxiety ${ }^{4,5}$ and depressive

*Address for correspondence: Marta Valdagno, Division of Psychiatry, Department of Molecular Medicine, University of Siena School of Medicine, Viale Bracci 1, Siena, Italy.

(Email marta.valdagno@gmail.com) disorders, ${ }^{4,5}$ especially during the maintenance phase, after achieving clinical remission from the most acute symptoms. In this context, telepsychiatry allows a more frequent and efficient monitoring, while decreasing the need for face-to-face sessions, and reducing costs for both the patient and the healthcare system.

Telepsychiatry may be accepted very cautiously in some cultures, especially those cultures that emphasize personal relationships. In such contexts, telepsychiatry may be perceived as a hindrance to communication, or a tool that is inadequate to establish an adequate therapeutic relationship. ${ }^{7,8}$ Yet, patients generally report good levels of satisfaction, possibly due to easy access to specialist care and reduced waiting time. ${ }^{4}$

Clinician satisfaction is less well evaluated, though acceptance was reported to be high in most studies. ${ }^{4}$ Interestingly, the degree of satisfaction was often related to the quality of the audio and video used. ${ }^{5}$

Telepsychiatry has been primarily studied as a tool to make a diagnosis or to provide short-term follow up, whereas large and controlled studies on the efficacy to deliver long-term psychotherapy and pharmacotherapy are still warranted.

The new generation's familiarity with technology and its widespread use as a means of communication makes this a more feasible possibility; on the other hand, the lack of valid scientific evidence limits the full applicability of this technique. Similarly, guidelines or 
standardized procedures that regulate the ethical and forensic implications are strongly needed.

Clearly, the protection of personal data, with the highest standards of safety for patients is a must. ${ }^{9}$ For instance, Hyler and Gangure ${ }^{10}$ outline a number of possible scenarios in which ethical issues can be breeched. They state that the electronic transmission of patient data has a far greater potential for infringement of privacy and confidentiality of private psychiatric information. Confidentiality must be maintained during videoconferences, as it is possible for outsiders to witness the consultation without being visible on the screen.

What other potentially risks are there?

- Alteration of the transmitted data due to a malfunction of the equipment

- Sudden malfunction of equipment or defect operation with interruption and/or discontinuities in the transmission of images

- Incorrect interpretation of data and/or diagnostic intervention and/or treatment from health care professionals

In our opinion the main targets that need to be achieved in this field include the following:

- Protecting patient privacy in order to ensure a high degree of trust

○ Ensuring the highest safety protection for computer records, computer networks, and wireless connections

○ Use of encryption techniques and electronic signatures in order to protect privacy, user identification, legal validity, and verification of electronic documents

- Definition of the legal responsibilities of the health operators involved

- Identification of obligations and responsibilities, particularly with regard to informed consent

- Method of authentication and authorization for access to services, with a clear definition of which type of information will be available for each category of operators (medical specialists, general practitioners, nurses, podiatrists, social assistants)

Few studies have evaluated telemedicine in Italy. For instance, Bertetto et $a l^{11}$ proposed guidelines for a telemedicine project in the Piedmont area. The authors highlighted the potential use of telemedicine as a means for new opportunities for links between different healthcare sites and between different healthcare levels (hospitals, emergency units, institutions, general practitioners).

The first Italian example of a study on the diagnostic reliability and feasibility of a telemedicine service via teleconferencing systems in psychiatry was conducted by De Luca et al in 2005. ${ }^{12}$ The authors evaluated 8 subacute patients by teleconferencing, using the expanded version of the Brief Psychiatric Rating Scale (BPRS) and an original instrument, a toolbox called Panama Emergency Psychiatric Scale (PERPS), to summarize urgency, severity, treatment strategy, and need for involuntary commitment. The evaluations were compared with those made by "control" psychiatrists who performed a normal face-to-face examination using the same questionnaires. The results showed almost no differences in the 2 groups for certain BPRS factors and for all PERPS items, suggesting the feasibility and reliability of psychiatric evaluations of mixed subacute patients via a Web-based teleconferencing system.

Our experience with regard to the use of telepsychiatry is limited to the pharmacotherapy or psychotherapy follow-up of patients living far from our University Mental Health Center or to the follow-up treatment of patients who are reluctant to leave their homes because of disease related factors, ie, patients with obsessive compulsive disorder who are unable/unwilling to leave their homes and/or come to a healthcare facility. However, no initial intake/diagnostic evaluation has been made via telepsychiatry yet. Although all cases that we have treated to date are patients who were previously seen in our inpatient or outpatient facilities, it is remarkable that all of them reported very high ratings of satisfaction and adherence to telepsychiatry treatment. We are now considering the possibility of extending this treatment to other populations, and are presently working at a specific protocol in this regard.

In conclusion, telepsychiatry is a new and growing field with excellent prospects and many potential clinical applications. However, more studies are needed to ensure a broader and more reliable validation of telepsychiatry, in terms of diagnostic reliability and therapeutic efficiency in the short and long term. Indeed, the development and the evaluation of standard protocols and practice guidelines are essential to a broader implementation. Likewise, focused and continuous training to increase the awareness of the benefits and risks of telepsychiatry are required. Among other benefits, this may be a way to help cultural acceptance of telepsychiatry both among patients and the medical staff.

In Italy, there are currently no reported developments of telemedicine services on a large scale, but only fragmented and experimental projects of uncertain duration in limited areas and not fully integrated into the health system. Many health providers trust the benefits of telemedicine, but several barriers hinder a wider use of telemedicine in Italy. For instance, most health services are delivered through the National Public Health System, whose large and relatively rigid structure often increases the resistance to change. However, the need for affordable, high quality, and alternative ways to deliver healthcare opens spaces that were unthinkable just a few years ago. 


\section{Disclosures}

Andrea Fagiolini is a grant recipient, consultant and/or speaker for the following institutions: Angelini, Lundbeck, Janssen, Bristol-Myers Squib, Pfizer, Eli Lilly, Astra Zeneca, and Otsuka. Drs. Valdagno, Goracci and di Volo do not have anything to disclose.

\section{REFERENCES:}

1. Wootton R, Yellowlees P, McLaren P. Telepsychiatry and e-Mental Health. London: Royal Society of Medicine Press Ltd., p. 3, 2003.

2. Manfredi L, Shupe J, Batki SL. Rural jail telepsychiatry: a pilot feasibility study. Telemed J E Health. 2005; 11(5): 574-577.

3. Rohland BM. Telepsychiatry in the heartland: if we build it, will they come? Community Ment Health J. 2001; 37: 449-459.

4. Hilty DM, Marks SL, Urness D, Yellowlees PM, Nesbitt TS. Clinical and educational telepsychiatry applications: a review. Can J Psychiatry. 2004; 49(1): 12-23.
5. Hilty DM, Luo JS, Morache C, Marcelo DA, Nesbitt TS. Telepsychiatry: an overview for psychiatrists. CNS Drugs. 2002; 16(8): 527-548.

6. Harley J, McLaren P, Blackwood G, Tierney K, Everett M. The use of videoconferencing to enhance tertiary mental health service provision to the island of Jersey. J Telemed Telecare. 2002; 8(Suppl 2): 36-38.

7. Shore JH, Savin DM, Novins D, Manson SM. Cultural aspects of telepsychiatry. J Telemed Telecare. 2006; 12(3): 116-121.

8. May C, Gask L, Atkinson T. et al: Resisting and promoting new technologies in clinical practice: the case of telepsychiatry. Soc Sci Med. 2001; 52(12): 1889-1901.

9. Hsiung RE. Therapy. London: W.W. Norton; 2002.

10. Hyler SE, Gangure DP. Legal and ethical challenges in telepsychiatry. J Psychiatr Pract. 2004; 10(4): 272-276.

11. Bertetto O, Lombardo M, Allocco M. Linee Guida per lo Sviluppo di un Progetto di Telemedicina. Politeia Edizioni, Beinasco (To); 2009.

12. De Luca V, Baldi G, Bernardini M, et al. New possibilities for psychiatric evaluations through Web-based (systems): a pilot study. Nuove possibilità di valutazioni psichiatriche con sistemi web-based: uno studio pilota. Rivista di Psichiatria. 2005; 40(5): 300-306. 